\title{
Prácticas usadas en los colegios de Bogotá para prevenir consumo de sustancias psicoactivas y agresión por estudiantes de secundaria y media
}

Practices Used in Schools in Bogotá to Prevent the Consumption of Psychoactive Substances and Aggression in Middle and High School Students

Práticas usadas nas escolas de Bogotá para impedir o uso de substâncias psicoativas e a agressão por estudantes do ensino médio e superior

\footnotetext{
Darío Maldonado** (iD) orcid.org/0000-0003-3544-208x

Catalina Salcedo*** (iD) orcid.org/0000-0002-4536-2166

Sandra García**** (iD orcid.org/0000-0003-4474-8603

Andrés Molano ${ }^{* * * * *}$ (D) orcid.org/0000-0002-2108-1685

Carolina Blanco****** (iD) orcid.org/0000-0001-6512-4408

Adriana Vargas ${ }^{* * * * * * *}$ (i) orcid.org/0000-0003-4665-8265
}

* Financiamiento: Fondo Nacional de Financiamiento para la Ciencia, la Tecnología y la Innovación -Francisco José de Caldas-Apoyo de la Secretaría de Educación de Bogotá.

** Ph. D. en Economía. Profesor Asociado, Universidad de los Andes, Bogotá, Colombia. Correo electrónico: dmaldonadoc@uniandes.edu.co

*** Magíster en Educación. Asistente graduada de la Universidad de los Andes, Colombia. Correo electrónico: c.salcedoa@uniandes.edu.co

**** Ph.D. en Política Social. Profesor Asociado, Universidad de los Andes, Bogotá, Colombia. Correo electrónico: sagarcia@uniandes.edu.co

***** Doctor en Educación y Desarrollo Humano. Profesor asociado de la Facultad de Educación. Universidad de los Andes, Bogotá, Colombia. Correo electrónico: aemll@nyu.edu

PH.D. in Architecture and Structural Design Profesora Asistente del Departamento de arquitectura de Ia Universidad de los Andes, Bogotá, Colombia. Correo electrónico: ac.blanco@uniandes.edu.co

****** Magíster en Políticas Públicas. Asistente graduada en la Universidad de los Andes, Bogotá, Colombia. Correo electrónico: am.vargasv@uniandes.edu.co 
Para citar este artículo: Maldonado, D. Salcedo, C., García, S., Molano, A., Blanco, C. y Vargas, A. (2O2O).

Prácticas usadas en los colegios de Bogotá para prevenir consumo de sustancias psicoactivas y agresión por estudiantes de secundaria y media. Revista Colombiana de Educación, 1(79)., 62-84. https://doi. org/10.17227/rce.num79-9851

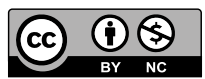

\section{Resumen}

Este artículo de investigación presenta resultados de un estudio sobre las prácticas usadas por los colegios de Bogotá para enfrentar los riesgos de agresión (física y verbal) y consumo de sustancias (alcohol, cigarrillo y drogas). En la investigación se usaron datos cuantitativos de una encuesta representativa a colegios de la ciudad y datos cualitativos de grupos focales con docentes y directivos docentes. Consideramos cuatro tipos de estrategias: punitivas, normativas, pedagógicas y espaciales. Se encuentra que, para el caso de la agresión, los cuatro tipos de estrategias son usadas. Los rectores reportan un mayor uso de estrategias pedagógicas y normativas que punitivas o espaciales, pero los docentes reportan un uso muy similar de las cuatro estrategias. Los resultados cualitativos se usan para discutir alternativas de política, la clasificación de estrategias y cómo, en muchos casos, esta clasificación tiene matices.
Recibido: 12/04/2019

Evaluado: 06/07/2019

\section{Palabras clave}

educación media; prácticas educativas; colegios; espacios escolares; edificios escolares; agresión; abuso de sustancias

\section{Keywords}

high schools; educational practices; school space; school buildings; aggression; substance abuse

\begin{abstract}
This article presents results from a research about the practices used by schools in Bogotá to deal with the risks of aggression (physical and verbal) and substance use (alcohol, cigarettes and drugs). The research used quantitative data from a representative survey of schools in the city and qualitative data from focus groups with teachers and school principals. We consider four types of strategies: punitive normative, pedagogical, and spatial. It is found that, in the case of aggression, all four types of strategies are used. According to school principals, pedagogical and normative strategies are more common than punitive and spatial ones; according to teachers all four strategies are equally used. The qualitative results are used to discuss policy alternatives, the classification of strategies and how, in many cases, this classification has nuances.
\end{abstract}

\section{Resumo}

Este artigo de pesquisa apresenta os resultados de um estudo sobre as práticas utilizadas pelas escolas de Bogotá para enfrentar os riscos de agressão (física e verbal) e uso de substâncias (álcool, cigarro e drogas). A pesquisa utilizou dados quantitativos de uma pesquisa representativa das escolas da cidade e dados qualitativos de grupos focais com professores e reitor da escola. Consideramos quatro tipos de estratégias: punitiva, normativa, pedagógica e espacial. Verifica-se que, no caso de agressão, todos os quatro tipos de estratégias são utilizados. Os diretores relatam um uso maior de estratégias pedagógicas e normativas do que punitivas ou espaciais, mas os professores relatam um uso muito semelhante das quatro estratégias. Os resultados qualitativos são usados para discutir alternativas de políticas, a classificação de estratégias e como, em muitos casos, essa classificação possui nuances.

\section{Palavras-chave}

ensino médio; práticas educacionais; escolas; espaços escolares; prédios escolares; agressão; abuso de substâncias 


\section{Introducción}

La atención al desarrollo social y emocional de los adolescentes constituye uno de los retos para la política pública y para las distintas instituciones que atienden a este grupo etario (Unicef, 2011). Organizaciones internacionales han mostrado la importancia de esta dimensión del desarrollo como una de las necesidades para romper ciclos de transmisión intergeneracional de la pobreza (Beniell et ál., 2016; OMs, 2004; Unicef, 2011). La política pública nacional colombiana y la política local de Bogotá han considerado este reto como una de sus prioridades (Chaux, 2009; Congreso de la República de Colombia, 2013; De Salvador et ál., 2011). Aunque cada vez hay mayor conciencia sobre el tema, la tarea de entender cómo puede la sociedad asumir este reto para potenciar el desarrollo social y emocional de la población colombiana apenas comienza.

En atención a esta necesidad, en este trabajo indagamos por las acciones que se están llevando a cabo en los colegios de Bogotá para potenciar el desarrollo social y emocional de los jóvenes que cursan secundaria. Partimos de la idea de que las estrategias que se asumen en los colegios para afrontar los riesgos a los que los estudiantes se ven expuestos puede ser determinante de cómo estos mismos riesgos afectan el desarrollo de los estudiantes. Nos concentramos en las acciones realizadas en los colegios porque constituyen un espacio fundamental en donde los estudiantes enfrentan riesgos y oportunidades en contexto.

Nos enfocamos en dos conductas de riesgo a las que están altamente expuestos los jóvenes y adolescentes, que son el consumo de sustancias y la agresión (Maldonado et ál., 2019). Estas dos conductas ponen en riesgo el desarrollo cognitivo y socioemocional de los jóvenes y por esta razón los colegios deben buscar activamente la reducción de su incidencia (Hertz et ál., 2015; Jefferis et ál., 2005; Mcleod et ál., 2004; Moore et ál., 2007; Rehm et ál., 2007; Silins et ál., 2014; Viner y Taylor, 2007; Elders et ál., 2014). Las dos conductas están relacionadas con la propensión a tomar riesgos, la propensión a seguir los comportamientos de sus pares y a la sensibilidad a la presión del grupo social (Currie et ál., 2012). En el caso del uso o consumo de alcohol, tabaco y otras sustancias, esta disposición está relacionada con la tendencia a la experimentación sin medir las consecuencias a largo plazo de sus acciones (Unicef, 2011; Romer, 2010).

Por su parte, la agresión se origina principalmente en la ausencia de herramientas para resolver conflictos de forma pacífica que llevan a procesar información de forma sesgada y derivan en comportamientos como la incapacidad de negociar, el alarde de poder y el exceso de competencia (Salcedo y Trujillo, 1997; Spillmann y Spillmann, 1991; Moeller, 2001). La reducción de la incidencia de estas conductas implica, entonces, desarrollar habilidades que permitan fortalecer la forma en que se procesan el conocimiento y las 
emociones, se sopesan distintos elementos que deben ser tenidos en cuenta a la hora de actuar y se toman decisiones; por ejemplo, el auto-conocimiento, la postergación de la gratificación o la capacidad de resistir a la presión de pares (Molano, Harker y Rodríguez, 2014).

El objetivo principal de este trabajo es conocer las estrategias utilizadas por los establecimientos educativos en Bogotá para prevenir y enfrentar el problema de consumo de sustancias y la agresión entre estudiantes de secundaria. Las dos conductas son susceptibles de ser prevenidas y desde el colegio es posible llevar a cabo acciones en este sentido; existe evidencia que soporta esta idea (Jones y Bouffard, 2012). Para ello, usamos dos fuentes de información. Primero, usamos los datos de una encuesta realizada a docentes y rectores de una muestra representativa de colegios oficiales y no oficiales de Bogotá. Segundo, usamos datos cualitativos recolectados a partir de grupos focales con docentes y directivos docentes de un subconjunto de estos mismos colegios. En ambos instrumentos de recolección de información (las encuestas y los grupos focales) se les preguntó a los participantes por las estrategias y los programas que usan en sus colegios para enfrentar estos riesgos.

Usamos un marco conceptual en el que se clasifican estas estrategias en cuatro categorías: punitivas, normativas, pedagógicas y espaciales. Las estrategias corresponden con formas diferentes de enfrentar los comportamientos de riesgo por parte de estudiantes en los colegios. Las tres primeras categorías difieren en el énfasis que hacen en los distintos actores y en los objetivos que se quieren lograr con su aplicación. El énfasis de las categorías punitiva y normativa está en el comportamiento mismo, el objetivo es evitar que se repita el comportamiento (McClod y Wachtel, 2003; Vaandering, 2014). La categoría pedagógica hace énfasis en la importancia de usar la comprensión por parte de las personas involucradas, de las motivaciones y consecuencias del comportamiento para generar desarrollo de competencias y habilidades que puedan ser usadas más adelante cuando el joven esté involucrado en situaciones similares y requiera tomar decisiones de cómo comportarse en esas situaciones (Bravo, 2008). La cuarta categoría (espacial), tiene en cuenta la importancia del espacio físico del colegio para prevenir los comportamientos de riesgo por parte de los estudiantes (Astor y Meyer, 2001; Behre et ál., 2001; LaRusso et ál., 2009); esta categoría, es menos usual en la literatura y solo recientemente ha empezado a aparecer como un posible enfoque en la prevención de riesgos en población escolar. Las cuatro categorías también difieren en los agentes que tienen en cuenta, las prácticas punitivas y normativas tienden a centrarse solamente en la persona directamente involucrada en el comportamiento (el consumidor o el agresor) mientras que las prácticas pedagógicas y espaciales se centran en otro tipo de agentes (por ejemplo, afectados y observadores). 
Estas estrategias no solo difieren en sus características sino también en su efectividad; Skiba y Losen (2016), Skiba y Peterson (1999) muestran que las estrategias punitivas son menos efectivas que las estrategias normativas y pedagógicas. Pero además existe evidencia de que las estrategias punitivas tienen un alcance muy limitado o incluso pueden exacerbar ciertos comportamientos como la violencia en el colegio (Hynman y Perone, 1998; Losen y Martinez, 2013; Skiba y Losen, 2016; Skiba y Peterson, 1999) por lo que es importante conocer el alcance del uso de las distintas estrategias. Sobre las estrategias espaciales apenas empieza a haber evidencia por lo que no es posible decir mucho sobre su efectividad.

Nuestros resultados muestran que los distintos tipos de estrategias son usadas por los colegios de la ciudad con pocas diferencias entre colegios oficiales y no oficiales. Mostramos que hay un uso casi universal de estrategias pedagógicas y normativas; el uso de estrategias punitivas y espaciales es menor, pero su uso sigue siendo alto. Ante la evidencia de la baja efectividad de las estrategias punitivas (Skiba y Losen 1999; Skiba y Peterson, 1999) y de que, incluso, pueden tener efectos negativos (Hynman y Perone, 1998) surge la pregunta por la posibilidad de generar transformaciones para que, en los colegios, el uso de estas estrategias sea menos frecuente. Los resultados también sugieren que el uso de estrategias relacionadas con el manejo de los espacios puede ser potenciado.

La estructura de este artículo es la siguiente. Esta primera sección es la introducción. La segunda sección, establece el marco conceptual para la categorización de las estrategias usadas en los colegios. La tercera, explica las fuentes de información y los métodos usados. La cuarta, resume los resultados del análisis y la quinta, concluye.

\section{Marco conceptual}

Las estrategias utilizadas para enfrentar y prevenir las conductas de riesgo de los jóvenes pueden ser muy diversas, no solamente en términos de características de las mismas medidas, sino también en cuanto al tipo de consecuencias que estas traen para los estudiantes involucrados. Para este trabajo, las estrategias se clasificaron en cuatro categorías, según su aproximación: punitivas, normativas, pedagógicas y espacial.

El enfoque punitivo, propuesto por McCold y Wachtel (2003) desde la perspectiva de la disciplina social, se caracteriza por tener un alto nivel de control (establecimiento de límites claros en el comportamiento) y un bajo apoyo al individuo (poca ayuda y soporte). En este enfoque se tiende a estigmatizar negativamente a la persona que ha cometido una falta, se penaliza su actuar y no se ofrecen oportunidades de reparación (Wachtel, 1999). 
Como su nombre lo indica, el enfoque normativo, parte del ámbito legal o normativo. En el caso colombiano, el artículo 41 del Decreto 1965 de 2013 y el Manual R.E.M.A de 2011 (Red de Mutua Ayuda), ofrecen una ruta mínima de atención con las acciones que cada establecimiento educativo debe desarrollar cuando se presentan situaciones de agresión y consumo de sustancias, respectivamente. En estos documentos se enfatiza la importancia de plantear mecanismos que fortalezcan los factores protectores en dichos ámbitos.

Por su parte, las estrategias con enfoque pedagógico abarcan la constitución de escenarios curriculares de formación e interacción entre prácticas, valores y conocimientos (Bravo, 2008). Así, se consideran las acciones orientadas al desarrollo de competencias de los estudiantes por medio de actividades.

Por último, el enfoque espacial responde a literatura reciente que apoya la creación de estrategias orientadas a identificar "espacios no reclamados", con el fin de trabajar con los miembros de la comunidad para apropiarse de los espacios e implementar acciones conducentes a la mitigación de riesgos y promoción de desarrollo de habilidades socioemocionales. Se entiende por "espacios no reclamados" los lugares como el patio de recreo, el comedor o cafetería escolar, los baños y corredores (Astor y Meyer, 2001; LaRusso et ál., 2009) en los cuales se presentan frecuentemente conflictos y se generan relaciones entre pares (Behre et ál., 2001).

\section{Datos y metodología}

Usamos dos fuentes de información para el presente trabajo. Primero, empleamos datos cuantitativos recogidos en campo en una muestra representativa de colegios oficiales y no oficiales de Bogotá que atienden principalmente estudiantes pertenecientes a hogares clasificados como de estratos bajo y medio (estratos 1 a 4 en la terminología oficial colombiana). En estos colegios se recolectaron datos de estudiantes de grados 9, 10 y 11 , profesores de estos tres grados y rectores. Para este trabajo se usan solamente los datos de docentes y rectores. Segundo, utilizamos datos cualitativos derivados de ejercicios de grupos focales con una submuestra de docentes y rectores que participaron en la encuesta.

\section{Datos cuantitativos}

La encuesta a docentes buscaba recoger información sobre conductas de riesgo de los estudiantes e información sobre estrategias y programas que se usan en los mismos colegios para enfrentar y prevenir estas problemáticas. La recolección de información la hizo un grupo de profesionales entrenados por 
los investigadores principales del estudio que visitaban los colegios para ese fin. Los investigadores principales también hicieron parte de la recolección de información. ${ }^{1}$ La muestra tiene 95 colegios, en tres de ellos visitamos la jornada de la mañana y la de la tarde; por esto hablamos de 98 colegios porque, en la práctica, en Bogotá el funcionamiento de un colegio en la mañana y en la tarde puede ser muy diferente, ya que la planta docente es distinta. En total 261 docentes y 94 rectores respondieron las encuestas.

La encuesta a docentes y directivos docentes indagaba sobre la ocurrencia o no de los siguientes comportamientos por parte de los estudiantes: consumo de alcohol, consumo de cigarrillo, consumo de drogas, agresión física entre estudiantes y agresión verbal entre estudiantes. Con respecto a las preguntas sobre el tipo de estrategias utilizadas en caso de conductas de riesgo, la encuesta preguntaba a los docentes y a los rectores para cada tipo de conducta (agresión física, agresión verbal, consumo de cigarrillo, consumo de alcohol, consumo de droga), con qué frecuencia se aplicaba cada una de las estrategias, de una lista de diferentes prácticas o acciones posibles (12 para el caso de agresión, 15 para el caso de consumo de alcohol y droga, y 13 para el caso de consumo cigarrillo). La respuesta estaba en la forma de una escala Likert con 5 opciones: Nunca, Pocas, A veces, Casi Siempre, Siempre.

Las estrategias incluidas en estas preguntas fueron adaptadas del cuestionario escolar "Healty and Safe School Environment" del Centro de Prevención de Enfermedades de Estados Unidos² ${ }^{2}$ así como de la revisión de la normativa colombiana y de Bogotá sobre la forma en que los colegios deben actuar en estos casos.

Adicionalmente, la encuesta a los rectores indagó por ciertas estrategias en espacios físicos. A diferencia de las preguntas sobre acciones frente a consumo y agresión, las preguntas sobre estrategias en el espacio físico no hacen referencia a una conducta en particular. Es decir, no se puede separar si la estrategia se usa para mitigar el riesgo de agresión o de consumo.

La tabla 1 muestra las diferentes acciones incluidas en la encuesta, clasificadas de acuerdo con las categorías descritas en la sección 2.

1 El proyecto fue aprobado por el comité de ética de la Universidad de los Andes, acta 569 de 2016 de agosto de 2016. Los instrumentos también fueron revisados y aprobados por la Secretaría de Educación del Distrito.

2 https://www.cdc.gov/healthyyouth/data/shpps/files/questionnaires/envl2014questionnaire.pdf 


\section{Tabla 1.}

Estrategias usadas por los colegios para enfrentar los problemas de agresión física y verbal y de consumo de cigarrillo, alcohol y marihuana

\begin{tabular}{|c|c|c|}
\hline \multicolumn{2}{|c|}{ Tipo } & Práctica \\
\hline \multirow{5}{*}{\multicolumn{2}{|c|}{ Punitivas }} & $\begin{array}{l}\text { Obligados a participar en algún programa educativo o de } \\
\text { apoyo }\end{array}$ \\
\hline & & $\begin{array}{l}\text { Retirados de participar en actividades extracurriculares o } \\
\text { torneos deportivos }\end{array}$ \\
\hline & & Suspendidos del colegio \\
\hline & & Expulsados del colegio \\
\hline & & Trasladados a otro colegio \\
\hline \multirow{8}{*}{\multicolumn{2}{|c|}{ Normativas }} & $\begin{array}{l}\text { Remitidos a hablar con un consejero de la institución (director } \\
\text { de curso u orientador) }\end{array}$ \\
\hline & & Remitidos a un directivo de la institución (rector o coordinador) \\
\hline & & $\begin{array}{l}\text { Instados a firmar el libro o se les ha hecho anotación en el } \\
\text { observador/anecdotario u otro tipo de registro del estudiante }\end{array}$ \\
\hline & & Citados sus padres o acudientes \\
\hline & & Remitidos al comité escolar de convivencia \\
\hline & & Remitidos a la Empresa Prestadora de Salud (EPS) * \\
\hline & & Remitidos a las autoridades legales ** \\
\hline & & $\begin{array}{l}\text { Reportados al sistema de alertas de la Secretaría de Educa- } \\
\text { ción Distrital *** }\end{array}$ \\
\hline \multirow{2}{*}{\multicolumn{2}{|c|}{ Pedagógicas }} & Sancionados pedagógicamente o con sanciones reparativas \\
\hline & & $\begin{array}{l}\text { Motivados, pero no obligados, a participar en algún programa } \\
\text { educativo o de apoyo }\end{array}$ \\
\hline \multirow{12}{*}{ Espacios } & \multirow{4}{*}{$\begin{array}{l}\text { Vigilancia } \\
\text { adentro del } \\
\text { colegio }\end{array}$} & Hay personal en los corredores o pasillos durante las clases \\
\hline & & Hay personal en los corredores o pasillos entre clases \\
\hline & & Hay personal en los baños \\
\hline & & Hay personal en los patios y otros espacios abiertos \\
\hline & \multirow{4}{*}{$\begin{array}{l}\text { Vigilancia en } \\
\text { el exterior del } \\
\text { colegio }\end{array}$} & Su colegio utiliza policías \\
\hline & & Su colegio utiliza vigilantes sin armas \\
\hline & & Su colegio utiliza vigilantes con perros \\
\hline & & Su colegio utiliza vigilantes con armas \\
\hline & \multirow{4}{*}{$\begin{array}{l}\text { Uso de } \\
\text { dispositivos }\end{array}$} & $\begin{array}{l}\text { Este colegio utiliza cámaras de seguridad dentro de los } \\
\text { edificios }\end{array}$ \\
\hline & & Utiliza cámaras de seguridad fuera de los edificios \\
\hline & & Utiliza detector de metales \\
\hline & & $\begin{array}{l}\text { Utiliza dispositivos de comunicación para propósitos de } \\
\text { seguridad }\end{array}$ \\
\hline \multicolumn{3}{|c|}{$\begin{array}{l}\text { *Válido solo para consumo de cigarrillo, alcohol y SPA } \\
\text { ** Válido solo para consumo de alcohol y SPA } \\
\text { *** Solo para consumo de cigarrillo, alcohol y SPA }\end{array}$} \\
\hline \multicolumn{3}{|c|}{ Fuente: elaboración propia. } \\
\hline
\end{tabular}


Además de las estrategias para enfrentar situaciones de consumo y agresión nos interesamos también por los programas que tienen los colegios para prevenir las conductas de riesgo. Para esto la encuesta inclúa una lista de posibles programas que los colegios usan habitualmente, así como su percepción sobre la efectividad de los mismos. Entre estos se incluyeron programas específicos para la prevención de agresión y de consumo, así como programas que no necesariamente tienen como objetivo principal prevenir estos riesgos pero que pueden incidir positivamente (por ejemplo, apadrinamiento entre estudiantes o educación en valores). En el análisis se incluyen las respuestas que dan docentes y rectores a los dos tipos de programas para obtener la comparación (entre programas que buscan incidir directamente en las conductas de riesgo y programas con otros objetivos).

\section{Datos cualitativos}

Para la realización de los grupos focales, en el momento de responder las encuestas cuantitativas, a todos los docentes les pedimos autorización para volverlos a contactar para invitarlos a la realización de los grupos focales. Luego de la invitación se hizo una reunión con los docentes interesados en la que les mostramos resultados sobre la incidencia de los riesgos de consumo y agresión y los lugares más frecuentes en que estos ocurren en el colegio (Maldonado et ál., 2019). Tras esa presentación, los docentes se distribuyeron en grupos con personas del equipo de investigación y de la Secretaría de Educación para responder dos preguntas. Primera, ¿qué tan parecido es su colegio a los datos presentados? Segunda, ¿cuáles son las intervenciones llevadas a cabo en cada institución para enfrentar estos riesgos? Estos grupos focales fueron grabados y las grabaciones se emplearon para hacer relatorías detalladas de las reuniones.

En los ejercicios con docentes y directivos no se dispuso de la clasificación de las estrategias; más bien se facilitó una conversación en la que ellos compartieron las estrategias y gracias a las grabaciones y las relatorías de esas sesiones se hizo la clasificación. Esta última fue realizada teniendo en cuenta el criterio de los investigadores y los relatos que docentes y directivos docentes hacen sobre ellas. Con la lectura de las relatorías y los audios de los grupos focales, tres personas diferentes del grupo de investigadores clasificaron las estrategias mencionadas por los participantes en las cuatro categorías de la Tabla 1. El ejercicio de clasificación se hizo atendiendo al lenguaje usado por los participantes para saber si en efecto la clasificación corresponde con la forma en que los docentes ven las estrategias. 


\section{Resultados}

\section{Resultados cuantitativos}

La tabla 2 muestra la proporción de colegios en donde son implementados los distintos tipos de estrategias de acuerdo con los reportes de docentes y rectores para cada una de las cinco conductas de riesgo consideradas (agresión física, agresión verbal, consumo de cigarrillo, consumo de alcohol y consumo de drogas). En el caso de los reportes de los docentes, como en cada colegio teníamos varios docentes encuestados, se reporta el porcentaje de colegios en donde al menos un docente reporta la aplicación de esas estrategias. El panel de arriba de la tabla muestra las estadísticas de los reportes de los docentes y el de abajo las de los reportes de los rectores. En cada caso se muestran cifras para los 98 colegios y cifras separadas para colegios oficiales y no oficiales. Los cálculos se realizaron teniendo en cuenta solo los colegios en donde los docentes y los rectores reportaron que se presentan casos de estos dos tipos de riesgo. Esto descarta la posibilidad de que, en los cálculos, se confunda reportes de que no se usan las estrategias porque el colegio no presenta ese riesgo, con reportes de ausencia de la aplicación de la estrategia cuando hay casos en que lo podrían requerir.

Empezamos por ver qué dice la tabla respecto de las estrategias para enfrentar riesgos de agresión y consumo en el panorama general de los colegios de la ciudad, sin distinguir por la naturaleza de los colegios (primera parte de la tabla para los rectores y primera parte de la tabla para los profesores). Según el reporte de los rectores, en los casos de agresión física y verbal prevalecen las estrategias normativas y pedagógicas, por encima de las punitivas. Sin embargo, el uso de estrategias punitivas no deja de tener una incidencia alta $(74,5 \%$ y $88,6 \%$ de los rectores reportaron usar estrategias punitivas en los casos de agresión verbal y física respectivamente). Para el caso de consumo, el uso de estrategias punitivas es diferente según el tipo de sustancia, siendo más alto en el caso de consumo de SPA (91.7 \%) en comparación con alcohol (81.8 \%) o cigarrillo (71.4\%); en todos los casos son mayores los reportes del uso de las estrategias punitivas y normativas. En el reporte de los docentes, las diferencias son sustancialmente menores y resalta que la proporción de docentes que reporta uso de estrategias punitivas es mucho más alta que en el caso de los rectores. Estos patrones se mantienen para los colegios oficiales y para los colegios no oficiales.

Vale la pena anotar que hay diferencias sistemáticas entre los reportes de los docentes y de los rectores en particular para los riesgos de consumo de sustancias. En particular, que de acuerdo con los rectores el uso de estrategias punitivas es menos común que de acuerdo con los docentes y 
que el uso de estrategias normativas y pedagógicas es mayor. Esto se cumple para todos los colegios, incluso cuando se considera los colegios oficiales y no oficiales por separado.

\section{Tabla 2.}

Colegios que usan estrategias de cada tipo de acuerdo con el reporte de los profesores y los rectores

\begin{tabular}{|c|c|c|c|c|c|c|c|}
\hline & & & $\begin{array}{c}\text { Agresión } \\
\text { verbal }\end{array}$ & $\begin{array}{l}\text { Agresión } \\
\text { fisica }\end{array}$ & Cigarrillo & Alcohol & SPA \\
\hline \multirow{9}{*}{ 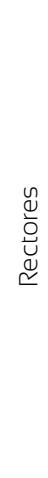 } & \multirow{3}{*}{ Todos } & Punitiva & $77,5 \%$ & $88,6 \%$ & $71,4 \%$ & $81,8 \%$ & $91,7 \%$ \\
\hline & & Normativa & $100 \%$ & $100 \%$ & $100 \%$ & $100 \%$ & $100 \%$ \\
\hline & & Pedagógica & $100 \%$ & $100 \%$ & $97,1 \%$ & $100 \%$ & $97,9 \%$ \\
\hline & \multirow{3}{*}{$\begin{array}{c}\text { No } \\
\text { oficiales }\end{array}$} & Punitiva & $64,9 \%$ & $89,3 \%$ & $50 \%$ & $66,7 \%$ & $100 \%$ \\
\hline & & Normativa & $100 \%$ & $100 \%$ & $100 \%$ & $100 \%$ & $100 \%$ \\
\hline & & Pedagógica & $100 \%$ & $100 \%$ & $75 \%$ & $100 \%$ & $85,7 \%$ \\
\hline & \multirow{3}{*}{ Oficiales } & Punitiva & $86,5 \%$ & $88,2 \%$ & $74,2 \%$ & $83,3 \%$ & $90,2 \%$ \\
\hline & & Normativa & $100 \%$ & $100 \%$ & $100 \%$ & $100 \%$ & $100 \%$ \\
\hline & & Pedagógica & $100 \%$ & $100 \%$ & $100 \%$ & $100 \%$ & $100 \%$ \\
\hline \multirow{9}{*}{ 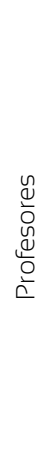 } & \multirow{3}{*}{ Todos } & Punitiva & $100 \%$ & $96,6 \%$ & $91,9 \%$ & $88,2 \%$ & $98,5 \%$ \\
\hline & & Normativa & $100 \%$ & $100 \%$ & $95,2 \%$ & $88,9 \%$ & $98,5 \%$ \\
\hline & & Pedagógica & $100 \%$ & $100 \%$ & $93,7 \%$ & $88,2 \%$ & $95,4 \%$ \\
\hline & \multirow{3}{*}{$\begin{array}{c}\text { No } \\
\text { oficiales }\end{array}$} & Punitiva & $100 \%$ & $91,7 \%$ & $83,3 \%$ & $90,9 \%$ & $100 \%$ \\
\hline & & Normativa & $100 \%$ & $100 \%$ & $88,9 \%$ & $92,9 \%$ & $100 \%$ \\
\hline & & Pedagógica & $100 \%$ & $100 \%$ & $88,9 \%$ & $90,9 \%$ & $100 \%$ \\
\hline & \multirow{3}{*}{ Oficiales } & Punitiva & $100 \%$ & $100 \%$ & $95,5 \%$ & $87,5 \%$ & $97,9 \%$ \\
\hline & & Normativa & $100 \%$ & $100 \%$ & $97,7 \%$ & $87,5 \%$ & $97,9 \%$ \\
\hline & & Pedagógica & $100 \%$ & $100 \%$ & $95,6 \%$ & $87,5 \%$ & $93,9 \%$ \\
\hline
\end{tabular}

Fuente: elaboración propia.

La tabla 3 reporta las respuestas que dan los rectores respecto al uso de estrategias espaciales. Más del $70 \%$ de los rectores reporta tener estrategias relacionadas con el espacio escolar. Específicamente, el 77,6 \% afirma tener vigilancia dentro de los espacios del colegio (personal en baños, corredores, patios y espacios abiertos); el 79,8 \% afirma utilizar vigilantes o policías; y el 83 \% afirma utilizar dispositivos de seguridad dentro o fuera del edificio (cámaras, detector de metales o radios de comunicación). El reporte de uso de estrategias espaciales es diferente entre colegios oficiales y no oficiales; los colegios oficiales utilizan menos vigilancia interna y 
más vigilancia externa y emplean más dispositivos que los colegios no oficiales. Los reportes de la aplicación de estas estrategias muestran que son menos comunes que el empleo de estrategias pedagógicas y normativas y de niveles similares al de estrategias punitivas.

Tabla 3.

Estrategias espaciales reportadas por los rectores

\begin{tabular}{lccc}
\hline & Vigilancia interna & Vigilancia externa & Uso de dispositivos \\
\hline Todos & $76,6 \%$ & $79,8 \%$ & $83 \%$ \\
No oficiales & $95,1 \%$ & $53,7 \%$ & $75,6 \%$ \\
Oficiales & $62,3 \%$ & $100 \%$ & $88,7 \%$ \\
\hline
\end{tabular}

Fuente: elaboración propia.

La tabla 4 muestra los reportes de los docentes y rectores sobre los programas con potencial de incidir en las conductas de riesgo presentes en los colegios. La tabla está organizada de forma que en el panel superior están los programas que no tienen como objetivo directo claramente identificable modificar conductas de riesgo; en el panel inferior, los que — de acuerdo con su denominación - sí tienen este objetivo. Dentro de cada uno de esos dos grupos, se organizaron los programas en orden de acuerdo con la proporción en que los rectores declaran tenerlos en sus colegios. Aunque no hay coincidencia exacta entre las proporciones de docentes y de rectores que dicen que en sus colegios se usa cada uno de los programas, el ordenamiento de los reportes de rectores y docentes tiende a coincidir. De acuerdo con el reporte de los rectores, los programas más comunes son los de programa de proyecto de vida (92,6\%), educación en valores (91,5\%), intervenciones para estudiantes con problemas disciplinarios (91,5 \%) y prevención consumo de sustancias (90,4 \%). Por su parte, los programas menos comunes de acuerdo con los rectores son apadrinamiento entre estudiantes (48,9\%) y capacitación de docentes en aula (50 \%). De acuerdo con los docentes, los programas más usados son los de educación en valores (78,5\%), de proyecto vida $(72,4 \%)$ y resolución de conflictos $(70,9 \%)$; y los programas menos usados son prevención de la deserción (31,8 \%), apadrinamiento de estudiantes (34,9\%) y capacitación de docentes en aula (36 \%).

El hecho que se destaca en la tabla 4 es que los programas que tienen como objetivo directo incidir en las conductas de riesgo de los estudiantes, relacionadas con agresión y consumo, son comunes y algunos de ellos son incluso más frecuentes que otro tipo de programas para problemas muy comunes en el sistema educativo como la deserción. Sin embargo, aún hay espacio de mejoramiento, particularmente en prevención de agresión y matoneo donde según el reporte de los rectores se encuentra presente 
en $75,5 \%$ de los colegios. Llama la atención la brecha entre el reporte de los docentes y rectores. Por ejemplo, mientras que el 90,4\% de los rectores reportan la presencia de programas de prevención de sustancias, en $62 \%$ de los colegios participantes, al menos un docente manifestó la existencia de este tipo de programas.

\section{Tabla 4.}

Programas usados en los colegios

\begin{tabular}{lcc}
\hline \multicolumn{1}{c}{ Programas } & Rectores & Profesores \\
\hline Programa de proyecto de vida/Orientación vocacional & $92,6 \%$ & $72,4 \%$ \\
Educación en valores & $91,5 \%$ & $78,5 \%$ \\
Prevención en deserción & $64,9 \%$ & $31,8 \%$ \\
Capacitación de docentes en aula & $50 \%$ & $36 \%$ \\
Apadrinamiento entre estudiantes & $48,9 \%$ & $34,9 \%$ \\
\hline Intervenciones para estudiantes con problemas disciplinarios & $91,5 \%$ & $70,9 \%$ \\
Prevención consumo sustancias & $90,4 \%$ & $62,1 \%$ \\
Derechos Humanos & $89,4 \%$ & $64,8 \%$ \\
Resolución de conflictos & $87,2 \%$ & $70,9 \%$ \\
Cátedra de paz & $87,2 \%$ & $64,4 \%$ \\
Mediación escolar entre pares & $83 \%$ & $56,3 \%$ \\
Prevención de agresión o matoneo & $75,5 \%$ & $60,5 \%$ \\
Currículo en resolución de problemas o habilidades sociales & $63,8 \%$ & $40,2 \%$ \\
Programa de manejo de disciplina & $64,9 \%$ & $44,1 \%$ \\
Reporte anónimo de problemas de seguridad & $66 \%$ & $36,8 \%$ \\
\hline Fuente: & & \\
\hline
\end{tabular}

Fuente: elaboración propia.

La tabla 5 muestra los programas empleados en colegios, separando por la naturaleza del colegio. El patrón de programas usados por los colegios oficiales y no oficiales difiere de forma importante entre ellos y del patrón general. Siguen siendo importantes en los dos grupos de colegios los de proyecto de vida, educación en valores, intervenciones para estudiantes con problemas disciplinarios y prevención consumo de sustancias. Sin embargo, entre los colegios oficiales son más frecuentes los programas de mediación escolar entre pares (92.5\% de acuerdo con los rectores) mientras que entre los colegios no oficiales son más frecuentes los de cátedra de paz (95.1 \% de acuerdo con los rectores) y derechos humanos (92.7\% de acuerdo con los rectores). En cuanto a los programas de prevención de consumo, llama 
la atención que no se encuentran diferencias entre colegios no oficiales y oficiales. En contraste, la presencia de programas de prevención de agresión o matoneo, así como la capacitación de docentes en aula es mayor en los colegios no oficiales en comparación con los oficiales (según el reporte tanto de docentes como de rectores).

\section{Tabla 5.}

Programas usados en los colegios según la naturaleza del colegio

\begin{tabular}{|c|c|c|c|c|}
\hline & \multicolumn{2}{|c|}{ Rectores } & \multicolumn{2}{|c|}{ Profesores } \\
\hline & $\begin{array}{l}\text { No } \\
\text { oficiales }\end{array}$ & Oficiales & $\begin{array}{c}\text { No } \\
\text { oficiales }\end{array}$ & Oficiales \\
\hline $\begin{array}{l}\text { Programa de proyecto de vida/Orientación } \\
\text { vocacional }\end{array}$ & $97,6 \%$ & $88,7 \%$ & $74,3 \%$ & $70,9 \%$ \\
\hline Educación en valores & $100 \%$ & $84,9 \%$ & $84,1 \%$ & $74,3 \%$ \\
\hline Prevención en deserción & $61 \%$ & $67,9 \%$ & $35,4 \%$ & $29,1 \%$ \\
\hline Capacitación de docentes en aula & $85,4 \%$ & $22,6 \%$ & $59,3 \%$ & $18,2 \%$ \\
\hline Apadrinamiento entre estudiantes & $53,7 \%$ & $45,3 \%$ & $45,1 \%$ & $27 \%$ \\
\hline $\begin{array}{l}\text { Intervenciones para estudiantes con } \\
\text { problemas disciplinarios }\end{array}$ & $92,7 \%$ & $90,6 \%$ & $78,8 \%$ & $64,9 \%$ \\
\hline Prevención consumo sustancias & $90,2 \%$ & $90,6 \%$ & $60,2 \%$ & $63,5 \%$ \\
\hline Derechos Humanos & $92,7 \%$ & $86,8 \%$ & $71,7 \%$ & $59,5 \%$ \\
\hline Resolución de conflictos & $85,4 \%$ & $88,7 \%$ & $68,1 \%$ & $73 \%$ \\
\hline Cátedra de paz & $95,1 \%$ & $81,1 \%$ & $74,3 \%$ & $56,8 \%$ \\
\hline Mediación escolar entre pares & $70,7 \%$ & $92,5 \%$ & $59,3 \%$ & $54,1 \%$ \\
\hline Prevención de agresión o matoneo & $82,9 \%$ & $69,8 \%$ & $64,6 \%$ & $57,4 \%$ \\
\hline $\begin{array}{l}\text { Currículo en resolución de problemas o } \\
\text { habilidades sociales }\end{array}$ & $78 \%$ & $52,8 \%$ & $48,7 \%$ & $33,8 \%$ \\
\hline Programa de manejo de disciplina & $78 \%$ & $54,7 \%$ & $59,3 \%$ & $32,4 \%$ \\
\hline Reporte anónimo de problemas de seguridad & $58,5 \%$ & $71,7 \%$ & $40,7 \%$ & $33,8 \%$ \\
\hline
\end{tabular}

Fuente: elaboración propia.

\section{Resultados cualitativos}

Las tablas 6 y 7 muestran las estrategias reportadas por docentes y directivos docentes que participaron en los grupos focales. En la tabla 6 se presentan aquellas estrategias que pueden ser clasificadas en uno de los enfoques descritos anteriormente (punitivo, normativo, pedagógico o espacial) y en la tabla 7 se presentan estrategias adicionales que no pueden ser clasificadas en ninguno de estos cuatro grupos. 


\section{Tabla 6.}

Estrategias normativas, punitivas, pedagógicas y espaciales mencionadas por docentes y rectores en los grupos focales

\begin{tabular}{|c|c|}
\hline \multirow{7}{*}{ Normativa } & 1. Cambio curricular en las asignaturas \\
\hline & 2. Proyecto de resolución de conflictos \\
\hline & 3. Comité de convivencia \\
\hline & 4. Acuerdos con alumnos involucrados en venta de SPA \\
\hline & 5. Llamado a padres de familia (casos puntuales) \\
\hline & 6. Reuniones con padres de familia \\
\hline & 7. Servicio social \\
\hline \multirow{2}{*}{ Punitivas } & 1. Acompañamiento docente en horas de receso \\
\hline & 2. Requisas a estudiantes (en las salidas pedagógicas) \\
\hline \multirow{20}{*}{ Pedagógica } & 1. Club lúdico \\
\hline & 2. Juegos de mesa en el receso \\
\hline & 3. Aulas en paz \\
\hline & 4. Proyecto de vida \\
\hline & 5. Visitas a la cárcel distrital \\
\hline & 6. Charlas con policía de infancia y adolescencia \\
\hline & 7. Proyecto de formación integral \\
\hline & 8. Programa mi colegio es territorio de sana convivencia \\
\hline & 9. Espacio plan de aula \\
\hline & 10. Proyecto de habilidades sociales \\
\hline & 11. Cátedra de formación ciudadana \\
\hline & 12. Proyecto de inmersión de investigación efectos SPA \\
\hline & 13. Charlas proyecto de vida \\
\hline & 14. Apoyo de orientación pedagógica \\
\hline & 15. Campañas de prevención \\
\hline & 16. Estrategias de prevención y afrontamiento en consumo de SPA \\
\hline & 17. Programa de media integral \\
\hline & 18. Talleres de emocionalidad y actividades propias con mujeres \\
\hline & 19. Proyecto Hermes \\
\hline & 20. Campañas visuales reflexivas \\
\hline \multirow{6}{*}{ Espacios } & 1. Rotación de salón en cambios de horario \\
\hline & 2. Patrulla escolar \\
\hline & 3. Apropiación de espacios \\
\hline & 4. Uso de cámaras de seguridad \\
\hline & 5. Acompañamiento y vigilancia en baños \\
\hline & 6. Apertura y cierre de baños en horas específicas \\
\hline
\end{tabular}




\section{Tabla 7.}

Otras estrategias mencionadas por docentes y rectores en los grupos focales

Estrategias orientadas a los docentes

1. Capacitación docente- pedagógica

Estrategias orientadas a la gestión

2. Ampliación de la jornada

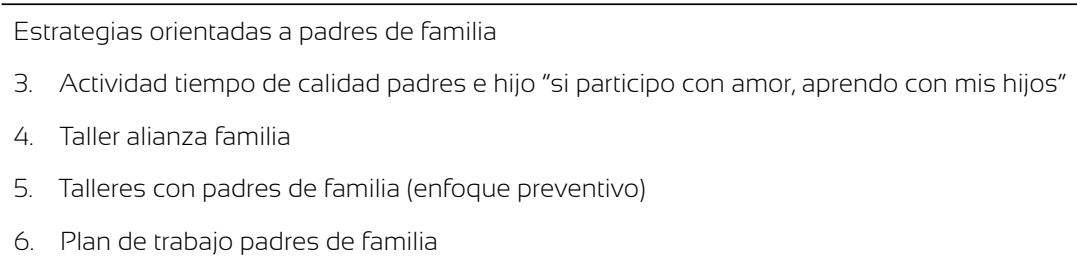

Estrategias orientadas a alianzas con otras entidades para la atención y prevención de conductas riesgosas

7. Caracterización de cursos para focalización de atención grupal

8. Alianzas con entidades relacionadas con la prevención del consumo de SPA

9. Alianzas con universidades y onc

10. Programa DARE

11. Bus interactivo

12. Programa Futuro Colombia

13. Alianzas con el Instituto Distrital de Recreación y Deporte (IDRD)

Fuente: elaboración propia.

Para el caso de las estrategias punitivas, los docentes y rectores hablaron solo de dos: acompañamiento docente en horas de receso y requisas a estudiantes en el contexto de las salidas pedagógicas. La primera se usa para reducir riesgos de agresión y la segunda, para reducir el riesgo de consumo. Llama la atención que en los grupos focales solo se hubieran mencionado dos estrategias en esta categoría. Las estrategias punitivas buscan demostrar a los estudiantes que los docentes y directivos docentes tienen un alto control de la situación y que, a su vez, con estas acciones se busca fomentar la disciplina y la instauración de límites para brindar un entorno sano para los estudiantes. La estrategia de acompañamiento docente en horas de receso tiene componentes espaciales; sin embargo, en el relato de los docentes aparecía como una estrategia punitiva.

Se identificaron cinco estrategias normativas. Se destaca el esfuerzo de las instituciones por aumentar el involucramiento parental con enfoque preventivo en la búsqueda que tanto padres como alumnos cumplan con los lineamientos institucionales y por afrontar los problemas del alumno con el acompañamiento de los padres, acudientes o grupo familiar de los alumnos. 
Dos de las estrategias categorizadas como normativas (acuerdos con alumnos involucrados en venta de sustancias psicoactivas y llamado a padres de familia en casos puntuales), también podrían ser considerada como punitivas. Para esto, las instituciones deberían obligar a los estudiantes a realizar acciones, pero según el relato que hicieron los participantes no se menciona si los acuerdos son resultado de un proceso pedagógico o de una imposición por parte del colegio.

Adicionalmente, en esta categoría también se encuentran dos estrategias que tienen un enfoque dual y que han sido estrategias normativas en su diseño, pero su implementación es pedagógica: cambio curricular y proyecto de resolución de conflictos. Con estas estrategias se ha buscado ofrecer herramientas y habilidades necesarias para que los estudiantes afronten los conflictos de una forma asertiva y así reduzcan los factores de riesgo de su entorno.

La gran mayoría de las estrategias mencionadas por los participantes pertenecen a la categoría de estrategias pedagógicas. En total, se mencionaron 20 estrategias diferentes en esta categoría. Las primeras 13 de este grupo aparecen en él porque su nombre deja ver que buscan que los estudiantes entiendan las dificultades asociadas con el consumo de sustancias y con la agresión; esto también se ve en los relatos que hacen los participantes de su uso. Las otras no entran de forma evidente en esta categoría, pero la forma en que los participantes las explican deja ver que su intención es pedagógica. Las estrategias pedagógicas expuestas por los participantes involucran aspectos lúdicos, académicos, acompañamiento especializado y parental, además de visualización de proyecto de vida. Las estrategias 1 y 2 de la tabla se centran en el aprovechamiento del tiempo libre. Estas tienen como objetivo ser una herramienta para fomentar los entornos protectores, reducir los factores de riesgo de los jóvenes y estimular la creatividad e imaginación de los estudiantes como parte de la formación integral.

Las estrategias 4 a 7 de la misma categoría, corresponden a acciones en las que los colegios aprovechan programas o instituciones de orden nacional. La mayoría de estos programas tienen enfoque preventivo e intervienen instituciones públicas y privadas en su ejecución. Las estrategias 8,9 y 10 tienen como objetivo brindar información a los jóvenes respecto a las consecuencias de tomar decisiones equivocadas (v.g. visitas a la cárcel), fomentar la construcción de proyecto de vida a través del conocimiento de casos de éxito de egresados o personas cercanas al entorno de los jóvenes y aumentar el conocimiento de los jóvenes respecto a los efectos en la salud y consecuencias legales respecto al consumo y tráfico de SPA, además de promover valores como la empatía y el autorreconocimiento focalizado en la construcción e identificación de objetivos personales. 
Por último, las estrategias pedagógicas de la 11 a la 16 reflejan el esfuerzo de las instituciones por diseñar planes y programas que aporten al crecimiento individual de los estudiantes $y$, a su vez, construyan de forma conjunta un entorno de sana convivencia en los espacios comunes por los alumnos.

Algunas de las estrategias de la categoría pedagógica tienen elementos específicos de prácticas restaurativas. Con base en lo expresado por los docentes y administrativos docentes, algunas de ellas, como las campañas visuales reflexivas, el programa Hermes, Mi colegio es territorio de sana convivencia y los talleres de emocionalidad con actividades propias para mujeres, promueven acciones restaurativas para resarcir el accionar equívoco de los alumnos. Ellos fomentan la creación de tejido social y de relaciones, la solución a problemas y conflictos, instauran límites y construyen de forma conjunta un entorno sano para los estudiantes.

La categoría "espacios" agrupa aquellas acciones que buscan mitigar algún tipo de conducta riesgosa al interior o exterior (más próximo) de las instituciones educativas. Se destacan seis intervenciones. Estrategias como la rotación de salones y apertura y cierre de baños en horas específicas son las más utilizadas por los colegios. Entre estas, los participantes le dieron especial atención a las dificultades del manejo de los baños que son lugares en donde reportan riesgo de agresión y consumo de forma frecuente; empero, reducir estos riesgos en estos espacios es difícil por el peligro de violar la privacidad de los estudiantes.

Finalmente, no todas las estrategias mencionadas se pueden clasificar en las cuatro categorías, estas aparecen en la Tabla 7. Se puede ver que entre ellas aparecen acciones orientadas a los docentes, a la gestión, a los padres de familia y alianzas con otras entidades. El factor común de las tres primeras es la construcción del conocimiento, pues se identifica que algunos de los principales actores involucrados en el desarrollo de los jóvenes (padres, acudientes y docentes) requieren de una periódica actualización en diversos temas.

Asimismo, se incluyen las alianzas con entidades estratégicas debido a que estas cuentan con personal idóneo y recursos para afrontar diversas situaciones a las que se encuentran expuestos los jóvenes. La mayoría de estas estrategias tienen un enfoque preventivo.

\section{Conclusión}

Este artículo usó información primaria obtenida en dos ejercicios de campo, uno de corte cuantitativo y otro de corte cualitativo, con docentes y rectores de Bogotá para documentar las prácticas que se usan en los 
colegios de la ciudad para enfrentar los riesgos de agresión y consumo de sustancias. Las estrategias se clasificaron en cuatro categorías: punitivas, normativas, pedagógicas y espaciales.

Se encontró que de forma general los cuatro tipos de estrategias son usadas en los colegios de la ciudad con algunas diferencias de acuerdo con el tipo de comportamiento. El uso de estrategias punitivas y normativas es menos común —-pero aún alto— que el uso de estrategias pedagógicas y espaciales.

Junto al uso de estrategias punitivas, normativas y pedagógicas se consideró el uso de estrategias espaciales. Estas tienen en cuenta que el espacio escolar puede incrementar o minimizar la ocurrencia de estos riesgos y sugiere la exploración a futuro de esta correlación entre el lugar y las actitudes de los estudiantes dentro del plantel educativo. Se encuentra que es común el uso de acciones como la vigilancia en el interior y el exterior del colegio y el uso de dispositivos como cámaras, detectores de metales, o dispositivos de comunicación. Finalmente, el trabajo también consideró los programas que reportan rectores y docentes para enfrentar estos riesgos.

De estos resultados se desprenden algunas discusiones e implicaciones interesantes. Primero, se ve un uso extendido de estrategias de los tres tipos distintos de las espaciales. Esto puede indicar que en los colegios hay una comprensión de los riesgos de consumo y agresión que los empodera para el uso de estrategias no punitivas. Las estrategias punitivas también son usadas, es posible que se deba a la necesidad de aprovechar complementariedades entre los tres tipos de estrategias o a límites en la efectividad de las normativas y pedagógicas. Sin embargo, surge la pregunta por la posibilidad de intervenciones que impulse el uso de acciones normativas y pedagógicas frente a las punitivas teniendo en cuenta que existe evidencia que muestra que estas difieren en su efectividad (Das et ál. 2016; Hynman y Perone, 1998; Losen y Martinez, 2013; Skiba y Losen. 2016; Skiba y Peterson, 1999).

Segundo, existe evidencia de que, en efecto, en los colegios hay un potencial para mejorar los comportamientos de los estudiantes (Dawkins et ál., 2015; Henessy et ál., 2015; MacArthur et ál., 2016; Onrust et ál., 2016; Pas et ál., 2015) pero hay fundamento para dudar de la idea de que las intervenciones son transferibles entre contextos distintos. Así como es importante entender el contexto en el que han crecido los estudiantes es también importante entender los contextos de los educadores y su conocimiento sobre el tipo de estrategias que pueden ser útiles para reducir comportamientos de riesgo de los estudiantes. Por ello, tener en cuenta resultados como los de este trabajo, acerca de las diferentes estrategias empleadas en colegios de Bogotá contribuye a la construcción de conocimiento general frente a riesgos de consumo y agresión. 
Tercero, surge una inquietud por las discrepancias en los reportes de docentes y rectores. Al respecto, se pueden plantear varias hipótesis para indagaciones futuras. Una primera conjetura, es la posibilidad de que en el colegio el rector tenga un mayor conocimiento detallado de eventos de riesgo por parte de los estudiantes; si este es el caso se debería considerar la necesidad de que en los colegios adopten políticas que hagan que todos los docentes conozcan la realidad de los estudiantes del colegio (sin violar su derecho a la privacidad y al debido proceso) de forma que los docentes se acerquen más a esas realidades y tengan más herramientas para apoyar su desarrollo social y emocional. La segunda posibilidad, contrariamente, es que detrás de la discrepancia haya un efecto de deseabilidad social que el rector siente que debe satisfacer al responder las preguntas, pero los docentes no lo sienten así. Una tercera hipótesis, es la existencia de normas implícitas y explícitas y que los reportes de los docentes y rectores están respondiendo a distintas formas de normas. Una última hipótesis, es que las expectativas de los rectores sobre las normas que se usan en las instituciones que dirigen corresponda con un ideal difícil de implementar en el contexto cotidiano. Las cuatro hipótesis sugieren intervenciones para apoyar las estrategias de los colegios para ofrecer mejores herramientas a sus estudiantes para su desarrollo. Pero también sugieren elementos que deben ser tenidos en cuenta en futuras investigaciones.

Finalmente, este trabajo presenta evidencia sobre diferentes estrategias utilizadas por colegios oficiales y no oficiales con la intención de prevenir y enfrentar comportamientos de riesgo por parte de los estudiantes. Sin embargo, el estudio no presenta la efectividad de estas estrategias. Futuras investigaciones deben centrarse en examinar en qué medida las estrategias utilizadas son efectivas o no, de manera que se puedan priorizar las acciones y maximizar los beneficios de las mismas.

\section{Referencias}

Astor, R. A. y Meyer, H. A. (2001). The conceptualization of violence-prone school subcontexts: Is the sum of the parts greater than the whole? Urban Education, 36(3), 374-399.

Behre, W. J., Astor, R. A. y Meyer, H. A. (2001). Elementary-and middle-school teachers' reasoning about intervening in school violence: An examination of violence-prone school subcontexts. Journal of Moral Education, 30(2), 131-153.

Berniell, L., de la Mata, D., Bernal, R., Camacho, A., Barrera-Osorio, F., Álvarez, F. y Vargas, J. (2016). RED 2016. Más habilidades para el trabajo y la vida: los aportes de la familia, la escuela, el entorno y el mundo laboral. Bogotá: Corporación Andina de Fomento (CAF). 
Bravo. N. H. (2008). Estrategias pedagógicas dinamizadoras del aprendizaje por competencias. Sinú. Colombia: Universidad del Sinú.

Bronfenbrenner, U. y Morris, P. A. (2007). The bioecological model of human development. Handbook of child psychology, 1.

Chaux, E. (2009). Citizenship competencies in the midst of a violent political conflict: The Colombian educational response. Harvard Educational Review, 79(1), 84-93.

Congreso de la República de Colombia (2013). Decreto 1965 de 2013. Sistema Nacional de Convivencia Escolar y Formación para el Ejercicio de los Derechos Humanos. Sexuales y Reproductivos y la Prevención y Mitigación de la Violencia Escolar. Bogotá.

Currie, C., Zanotti, C., Morgan, A., Currie, D., De Looze, M., Roberts, C. y Barnekow, V. (2012). Social determinants of health and well-being among young people. Health Behaviour in School-aged Children (HBSC) study: international report from the 2009/2010 survey. Copenhagen: WHO Regional Office for Europe.

Das, J. K., Salam, R. A., Arshad, A., Finkelstein, Y. y Bhutta, Z. A. (2016). Interventions for adolescent substance abuse: An overview of systematic reviews. Journal of Adolescent Health, 59(4), S61-S75.

Dawkins, J. D., Jenson, J. M., Catalano, R., Fraser, M. W., Botvin, G. J., Shapiro, V. y Rotheram-Borus, M. J. (2016). Unleashing the power of prevention. American Journal of Medical Research, 3(1), 39.

De Salvador, G. REMA Red de Ayuda Mutua: montaje de grupos de ayuda mutua en el contexto escolar. Intervención inicial para jóvenes y adolescentes con dificultades asociadas al uso de sustancias psicoactivas o violencias en contexto socio familiar con actividad escolar paralela. Bogotá: Bogotá positiva. https://repositoriosed.educacionbogota. edu.co/handle/001/1482

Elders, M. J., Perry, C. L., Eriksen, M. P. y Giovino, G. A. (1994). The report of the Surgeon General: preventing tobacco use among young people. American Journal of Public Health, 84(4), 543-547.

Hennessy, E. A. y Tanner-Smith, E. E. (2015). Effectiveness of brief schoolbased interventions for adolescents: a meta-analysis of alcohol use prevention programs. Prevention Science, 16(3), 463-474.

Hertz, M. F., Everett Jones, S., Barrios, L., David-Ferdon, C. y Holt, M. (2015). Association between bullying victimization and health risk behaviors among high school students in the United States. Journal of School Health, 85(12), 833-842.

Hyman, I. A. y Perone, D. C. (1998). The other side of school violence: Educator policies and practices that may contribute to student misbehavior. Journal of School Psychology, 36(1), 7-27. 
Jefferis, B. J., Power, C. y Manor, O. (2005). Adolescent drinking level and adult binge drinking in a national birth cohort. Addiction, 100(4), 543-549.

Jones, S. M. y Bouffard, S. M. (2012). Social and Emotional Learning in Schools: From Programs to Strategies and commentaries. Social policy report, 26(4), 1-33.

LaRusso, M. D., Jones, S. M., Brown, J. L. y Aber, J. L. (2009). School context and micro-contexts: The complexity of studying school settings. In LM. Dinella (Ed.), Conducting science-based psychology research in schools (pp. 175-197). Washington, D.C.: APA Books.

Losen, D. J. y Martinez, T. E. (2013). Out of school and off track: The overuse of suspensions in American middle and high schools. Ucla Ed \& Is. https://files.eric.ed.gov/fulltext/ED541735.pdf

MacArthur, G. J., Harrison, S., Caldwell, D. M., Hickman, M. y Campbell, R. (2016). Peer-led interventions to prevent tobacco, alcohol and/ or drug use among young people aged 11-21 years: a systematic review and meta-analysis. Addiction, 111(3), 391-407.

McCold, P. y Wachtel, T. (2003). In pursuit of paradigm: A theory of restorative justice. Paper presented at the XIII World Congress of Criminology (Vol. 10, p. 15).

Maldonado, D., Molano, A., García, S., Blanco, C. y Cuellar, W., (2019). Espacios no reclamados en la escuela: incidencia y prevalencia de riesgos para el desarrollo. Mimeo.

Moeller, T. G. (2001). Youth aggression and violence: A psychological approach. Routledge. London: Lawrence Erlbaum Associates, Inc.

Molano, A., Harker, A. y Rodríguez, G. I. (2014). Desarrollo de Habilidades Sociales y Emocionales para el Contexto Educativo Colombiano: Una Propuesta Inicial para la Educación Media. Bogotá: Documento preparado para el Ministerio de Educación Nacional.

Moore, T. H., Zammit, S., Lingford-Hughes, A., Barnes, T. R., Jones, P. B., Burke, M. y Lewis, G. (2007). Cannabis use and risk of psychotic or affective mental health outcomes: a systematic review. The Lancet, 370(9584), 319-328.

Onrust, S. A., Otten, R., Lammers, J. y Smit, F. (2016). School-based programmes to reduce and prevent substance use in different age groups: What works for whom? Systematic review and meta-regression analysis. Clinical Psychology Review, 44, 45-59.

Organización Mundial de la Salud (OMs) (2004). Promoción de la salud mental: conceptos, evidencia emergente y práctica. Departamento de salud mental y abuso de sustancias. Francia: Autor. 
Pas, E. T., Cash, A. H., O'Brennan, L., Debnam, K. J. y Bradshaw, C. P. (2015). Profiles of classroom behavior in high schools: Associations with teacher behavior management strategies and classroom composition. Journal of School Psychology, 53(2), 137-148.

Rehm, J., Shield, K. D., Joharchi, N. y Shuper, P. A. (2012). Alcohol consumption and the intention to engage in unprotected sex: Systematic review and meta-analysis of experimental studies. Addiction, 107(1), 51-59.

Romer, D. (2010). Adolescent risk taking, impulsivity, and brain development: Implications for prevention. Developmental Psychobiology: The Journal of the International Society for Developmental Psychobiology, 52(3), 263-276.

Salcedo F., A. yTrujillo T., J. (2014). Agresión, conflicto, violencia y paz. Universitas Humanística, 46 (46).

Silins, E., Horwood, L. J., Patton, G. C., Fergusson, D. M., Olsson, C. A., Hutchinson, D. M. y Coffey, C. (2014). Young adult sequelae of adolescent cannabis use: an integrative analysis. The Lancet Psychiatry, 1(4), 286-293.

Skiba, R. J. y Losen, D. J. (2016). From reaction to prevention: Turning the page on school discipline. American Educator, 39(4), 4.

Skiba, R. J. y Peterson, R. (1999). The dark side of zero tolerance: Can punishment lead to safe schools? The Phi Delta Kappan, 80(5), 372-382.

Spillman, K. y Spillman, K. (1991). La imagen del enemigo y la escalada de los conflictos. Revista Internacional de Ciencias Sociales, 127, 59-79.

United Nations Children's Fund (Unicef), (2011). Adolescencia una época de oportunidades. Estado mundial de la infancia 2011. https:// www.unicef.org/spanish/sowc2011/pdfs/SOWC-2011-Main-Report_ SP_02092011.pdf

Vaandering, D. (2014). Implementing restorative justice practice in schools: What pedagogy reveals. Journal of Peace Education, 11(1), 64-80.

Viner, R. M. y Taylor, B. (2007). Adult outcomes of binge drinking in adolescence: findings from a U.K. national birth cohort. Journal of Epidemiology \& Community Health, 61(10), 902-907.

Wachtel, T. (1999, 16-18 de febrero). Justicia restaurativa en la vida cotidiana: más allá del ritual formal. Conferencia Reconfigurando las Instituciones Australianas: Justicia Restaurativa y Sociedad Civil. The Australian National University, Canberra. https://www.iirp.edu/news/ justicia-restaurativa-en-la-vida-cotidiana-mas-alla-del-ritual-formal 